Aim To look at the effect of the consultant delivered care in comparison to the registrar delivered care on the clinical services, patient care, satisfaction and staff opinion.

Method All the patients attending our paediatric assessment unit between 1130 am and 2100 were assessed on three consecutive days for two weeks. The parameters compared were the length of time taken to make a clinical decision from the time the patient was seen, investigations performed, any readmissions, patient outcome, any significant incidents, patient satisfaction and nursing staff opinion. Results A total of 51 patients were analysed. 21 of them were seen by the consultants and 29 were seen by the registrars. The time taken for Consultants to make the clinical decision and management plan varied from 15 to 100 minutes with a median of 30 minutes and mean of 39.6 minutes. The registrars took 90 to 480 minutes for the clinical decision with a median time of 250 minutes and mean of 233 minutes. 4 (19\%) and $16(55 \%)$ patients seen by consultants and registrars had investigations respectively. 2 patients, seen by registrars, with Paediatric Early Warning Score of more than 4 were admitted after $280 \mathrm{~min}$. One of these patients needed HDU admission and was transferred to specialist hospital. Another patient was diagnosed to have sepsis and had acute deterioration. 16 (76\%) patients were discharged home and 5 (24\%) patients were admitted by consultants. In the group seen by registrars or speciality trainees, 10 patients (34.5\%) were sent home, 17 were admitted (59\%) and 2 were transferred out. There was one readmission from the group seen by the speciality trainees Patients' satisfaction survey was similar in both groups. Nursing staff in POAU felt there was quicker decision making and more precise plans when seen by the Consultants. Conclusion The consultants made faster decision, performed less investigations and discharged more patients home with no readmissions or clinical incidents.

\section{G132(P) USE OF NASAL CPAP IN INFANTS WITH BRONCHIOLITIS IN THE SOUTH OF ENGLAND: A MULTICENTRE, PROSPECTIVE, OBSERVATIONAL STUDY}

doi:10.1136/archdischild-2013-304107.144

${ }^{1,2}$ A Saha, ${ }^{2} \mathrm{E}$ Vamvakiti, ${ }^{2} \mathrm{M}$ Linney. ${ }^{1}$ Department of Paediatrics, Maidstone and Tunbridge Wells Hospitals NHS Trust, Maidstone, UK.; ${ }^{2}$ Department of Paediatrics, Western Sussex Hospitals NHS Trust, Worthing, UK

Aim Bronchiolitis is a common respiratory illness in childhood with 64 million cases of RSV bronchiolitis worldwide every year. In England, 2.8\% hospital admissions in children $<1$ year of age are due to RSV bronchiolitis with upto $5 \%$ of these patients going on to develop respiratory failure.

The Aim of this study was to review the use of nasal continuous positive airway pressure (nCPAP) in infants with bronchiolitis. A secondary aim was to identify predictive factors for CPAP failure resulting in endotracheal intubation and mechanical ventilation

Methods A prospective, multicentre, observational study was undertaken from 1November 2008 to 28February 2009. Seven Paediatric Units in the South of England participated in the study. Data was collected on indications for nCPAP, respiratory rate and blood gases prior to nCPAP, total number of days on nCPAP and length of hospital stay. Results A total of 51 infants with the clinical diagnosis of bronchiolitis required nCPAP during the study period. The main indications were increased work of breathing (47.0\%), apnoeas (39.2\%) and increasing oxygen requirements (23.5\%). Among them 16 were ex-preterm $(31.37 \%)$. Prior to nCPAP the mean respiratory rate was $63 / \mathrm{min}$ (range 28 to 120 ), mean oxygen saturations $85 \%(70$ to $98 \%$ ), mean pH 7.25(7.03 to 7.36) and mean PCO2 10.42(4.36 to 19.0). The average time on nCPAP was 2.6 days( 2 hours to 11 days) and the average length of hospital stay was 10.96 days (5 to 22 days). There were no reported dealths. 11 out of the 51 infants failed trial on nCPAP requiring intubation $(21.5 \%)$. The main indications were apnoeas $(45.45 \%), \mathrm{CO}_{2}$ retention $(36.36 \%)$ and increasing work of breathing (36.36\%). Subgroup analysis revealed that 7 out of the 11 infants requiring intubation were ex-preterm (63.6\%) and 8 had significant PCO2 rise (>8) prior to the trial of nCPAP (72.72\%).

Conclusions nCPAP has a good success rate in bronchiolitis. In our study, predictive factors associated with nCPAP failure were prematurity and high $\mathrm{CO}_{2}$ retention prior to trial of nCPAP. The authors recommend the availability of nCPAP facilities in all paediatric units and appropriate training of medical and nursing staff in its optimal use.

\section{G133(P) MANAGEMENT OF PROLONGED JAUNDICE IN NURSE-LED CLINICS - 10 YEARS OF A SAFE AND EFFICIENT SERVICE}

doi:10.1136/archdischild-2013-304107.145

1. 2M Hyrapetian, 'R J Hewitson, 'H Mackinnon. 'General Paediatrics, The Whittington Hospital NHS Trust, London, UK; 2 Paediatrics, London School of Paediatrics, London, UK

Aims Prolonged jaundice in babies is common and usually harmless but a very small number of cases have serious pathology, such as biliary atresia, in which early detection is vital. Many "well" babies with prolonged jaundice undergo extensive investigations with a very low yield of important positive results. Our aim was to assess the safety, efficiency and cost effectiveness of a nurse-led prolonged jaundice clinic which has been in operation at our district general hospital for ten years, performing minimal investigations compared to standard practise.

Methods We collected data retrospectively for all babies $<3$ months of age in whom a conjugated bilirubin level was measured at $\geq 14$ days of age (term) or $\geq 21$ days (preterm) from January-August 2011, excluding babies on the neonatal unit. We reviewed clinic proformas completed for each baby seen with prolonged jaundice, medical notes and hospital results system to establish whether the baby was managed in the nurse led prolonged jaundice clinic or elsewhere by doctors, investigations undertaken and clinical outcomes.

Results 91\% (176) of 194 babies were managed in the nurse led prolonged jaundice clinic; 5.6\% (10) had additional investigations which identified one case with significant pathology. Of the 9\% (18) of babies seen elsewhere by doctors (A\&E/outpatients/inpatients/ daycare), 77\% (14) had additional investigations. 3 babies had serious pathology including one case of biliary atresia which presented late (48 days old).

Conclusion For the last ten years we have managed the vast majority of babies with prolonged jaundice in our nurse-led clinic, successfully avoiding unnecessary, time-consuming and expensive investigations for well babies whilst promptly identifying cases with serious pathology.

Our district general hospital has recently merged with two local community health services to form an Integrated Care Organisation, providing a unique opportunity to move the nurse-led prolonged jaundice clinic into the community. Based on our well established model, such clinics could be conducted in local health centres by midwives or community paediatric nurses, supervised by a consultant paediatrician. This would allow further cost savings and be more convenient for families.

\section{G134(P) LUMBAR PUNCTURES IN CHILDREN: A SURVEY OF CURRENT PRACTISE}

doi:10.1136/archdischild-2013-304107.146

${ }^{1,2}$ D Son, ${ }^{2}$ R Roots. ${ }^{1}$ Paediatrics, Epsom and St Helier University Hospitals NHS Trust, Carshalton, Surrey, UK: ${ }^{2}$ Medical School, St George's University of London, London, UK

Aims Lumbar puncture (LP) is a common procedure in children used to diagnose infection and various neurologic processes. We want to survey current clinical practises, in terms of positioning and use of analgesia, in paediatric LPs.

Methods A survey questionnaire was designed for distribution to staff on the wards of 10 paediatric departments in one city. The 
survey contained questions about demographics, choice of position during LPs, reasons for that choice, use and frequency of analgesia, LP training background and demand for training. Questions about positions and pain relief were divided into different age groups.

Results A total of 84 questionnaires were completed, which demonstrated that the most common position being used in children under the age of 1 was the lateral recumbent position with neck flexion $(83 \%$ in newborns to 3 months and $59 \%$ in 3 months to 1 year). $61 \%$ of participants said this position was used to increase the interspinous distance, whilst $27 \%$ said is was used to best hold the child still. Sucrose was the most commonly used for of pain relief in children under one, however $39 \%$ of participants never, rarely or only sometimes used pain relief in this age group. $79 \%$ of participants would appreciate more training in this area.

Conclusion We demonstrated that a painful, uncomfortable and potentially dangerous position to hold children during LPs was the first choice in the majority of cases. We also demonstrated that the use of analgesia in general was either absent or poor. Further education of healthcare staff involved in this procedure is needed.

\section{G135(P) DEVELOPMENT OF CHILDREN AND YOUNG PEOPLE'S ASSESSMENT SERVICE STANDARDS AND A SUPPORTIVE PEER REVIEW PROCESS ACROSS A REGION}

doi:10.1136/archdischild-2013-304107.147

${ }_{1,2,3} \mathrm{M}$ Clements, ${ }^{1,3} \mathrm{~K}$ Evans, ${ }^{1,4} \mathrm{C}$ Upton. ${ }^{1}$ Strategic Network of Child Health and WellBeing, NHS East of UK, Cambridge, UK; ${ }^{2}$ Department of Paediatrics, West Suffolk Hospital NHS Foundation Trust, Bury St Edmunds, UK; ${ }^{3}$ Children and Young People programme, NHS Institute for Innovation and Improvement, Coventry, UK; ${ }^{4}$ Department of Paediatrics, Norfolk and Norwich University Hospital NHS Foundation Trust, Norwich, UK

Aims To develop a set of standards and a peer review process for Children and Young People's (CYP's) ambulatory care.

Methods Development of the standards involved reviewing national, regional and local policies/best practise. Questionnaires were circulated and focus groups facilitated with multidisciplinary staff involved in CYP ambulatory care across a region. Professionals challenged and tested the standards as they evolved. This included CYP and family feedback and enagement.

All 17 acute trusts involved in CYP acute care in the region performed a self-assessment using the assessment service standard tool and participated in an external peer review visit.

The visit started with informal discussions with staff and service users. The team walked the patient pathway. A whole-system professionals meeting, involving senior Managers/Executives, Clinicians/Senior Nursing team, Play Specialists and Commissioners concluded the visit. The local team presented their self-assessment and the visiting team feedback their findings leading to a discussion/action planning for next steps.

The process was evaluated.

Each organisation showcased the good practise identified at a celebration event.

Re-visits to all trusts are arranged to review progress and assess against the national standards for CYP in the emergency department. Results A supportive process for peer review for children and Young People's assessment services and emergency and urgent care, including comprehensive standards have been developed and evaluated.

A poster summarises feedback from Children, young people and families. Figure 1

Current practise for ambulatory care across a region has been reviewed and key findings from the visits including the variety of models of care, good practise and recommendations summarised and shared.

The visits were evaluated as supportive and useful in raising the profile of CYP within Trusts. The standards gave a focus for organisations to review their services and plan improvements.
There was evidence the standards had driven improvements and good practise identified in every unit.

Conclusion There is already evidence showing standards and peer review improves quality of care.

We have developed a processes and tools that can be used nationally for CYP's emergency and urgent care

These visits were not mandatory but $100 \%$ of Trusts participated in the self-assessment and peer review and booked re-visits.

\section{G136(P) IMMUNISATION OF HIV POSITIVE CHILDREN}

doi:10.1136/archdischild-2013-304107.148

${ }^{1}$ A Bailey, ${ }^{2}$ S Bandi. 'Department of Paediatrics, LNR Deanery, Leicester, UK; '2Leicester Children's Hospital, Leicester Royal Infirmary, Leicester, UK

Aims To determine if the immunisation schedules of thirty one children attending a tertiary paediatric HIV clinic in 2012 adhered to the Children's HIV association guidelines on immunisation in HIV positive children.

Methods We looked at the immunisation records of the thirty one children attending the tertiary paediatric HIV clinic. A standard proforma was used for data collection from the health care records, clinic letters and 'Red Book'. We also contacted the General Practice (GP) surgeries for those with inadequate information in the health records. Results Children were aged between four and sixteen years with a fairly even distribution between sexes. 25/31 children were born abroad. Children were classed as fully immunised if they were vaccinated according to UKguidelines. Overall $48 \%$ received complete primary and $38 \%$ received complete booster immunisations. $83 \%$ of immunisations were complete in UK born children compared with $28 \%$ in non-UK born children. We were unable to obtain immunisation information in $25 \%$ of children who were born abroad, there was no information regarding immunisation status in either health records or in GP surgery records.

Figure 1 details the immunisation details for all our children. In addition, Human Papilloma Virus (HPV) vaccine was given in $56 \%$ of eligible patients. $52 \%$ of patients received a BCG vaccine and all of them were born abroad. $48 \%$ received an annual influenza vaccine and $68 \%$ of children had received the Hepatitis $B$ vaccine.

Conclusions There is poor vaccination of children with HIV, especially those born abroad and there is an urgent need for strategies to be implemented in order to achieve better rates of immunisation. Recommendations include interface between hospitals and GP practises with improved access to immunisation records, reminder letters to GPs and families and possible opportunistic immunisation in hospitals.

\section{G137(P) HOW TO SAVE A SMILE!}

doi:10.1136/archdischild-2013-304107.149

${ }^{1} \mathrm{KG}$ Greenwood, 'DT Tuthill, ' $\mathrm{CP}$ Patel, ${ }^{2} \mathrm{EM}$ Hingston. 'General Paediatrics, Cardiff and Vale UHB, Cardiff, UK; ${ }^{2}$ Paediatric Dentistry, Cardiff and Vale UHB, Cardiff, UK

Introduction Dental caries is entirely preventable, yet remains the most common chronic disease of childhood- affecting $50 \%$ of children under five in Wales. It is caused by acid produced by the bacterial metabolism of dietary carbohydrates, particularly refined sugars. Frequent squeal include: pain, infection, poor aesthetics and permanent teeth damage. Basic standards of advice to prevent dental caries exist. ${ }^{1}$ Ensuring medical staff have the knowledge to advocate appropriate oral care, and know when to refer to dental colleagues could reduce the burden of dental disease.

Aims To audit knowledge of staff regarding dental health care in children against basic dental guidelines ${ }^{1}$.

Methods Structured questionnaires were designed by paediatricians and dentists and administered to healthcare staff. These were based upon: dietary advice including limitation of fruit juices, 